\title{
Use of Segregated Hepatocyte Scaling Factors and Cross-Species Relationships to Resolve Clearance Dependence in the Prediction of Human Hepatic Clearance
}

\author{
D. Hallifax and J.B. Houston \\ Centre for Applied Pharmacokinetic Research, Division of Pharmacy and Optometry, School of Health Sciences, Faculty of Biology, \\ Medicine and Health, Manchester Academic Health Science Centre, University of Manchester, Manchester, United Kingdom
}

Received October 29, 2018; accepted December 20, 2018

\begin{abstract}
Human and rat hepatocytes have a strong tendency to underpredict hepatic intrinsic clearance $\left(\mathrm{CL}_{\text {int }}\right)$ and the extent of underprediction increases with increasing observed $\mathrm{CL}_{\text {int. }}$ In this study, application of the log average rat hepatocyte-rat in vivo empirical scaling factor (ESF) of 4.2 to human hepatocyte prediction successfully removed bias but did not improve precision. An analogous method using individual drug rat ESFs only achieved marginal improvement in accuracy but not precision. A novel approach to resolve clearancedependent prediction, involving rat ESFs calculated for particular (order of magnitude) ranges of observed $\mathrm{CL}_{\text {int }}$ (log average range, 0.12-2.1) improved human prediction precision but only modestly reduced bias. However, rat in vivo $\mathrm{CL}_{\text {int }}$ was several-fold greater
\end{abstract}

than human in vivo $\mathrm{CL}_{\text {int }}$ and this was reflected in greater rat hepatocyte and microsome $\mathrm{CL}_{\text {int }}$, suggesting that rat metabolic enzymes are more efficient than their human counterparts, by several-fold. By applying the segregated rat ESFs followed by the human/rat $\mathrm{CL}_{\text {int }}$ ratio, which was consistent regardless of $\mathrm{CL}_{\text {int }}$ (log average 3.5), both accuracy and precision were improved, providing both a means of mitigating clearance dependence and reaffirming the potential role of rat hepatocytes for prediction of human metabolic $\mathrm{CL}_{\text {int }}$. These cross-species observations indicate that underprediction from human in vitro systems may be predominantly consequential of an intrinsic property of the in vitro system rather than individual drug properties.

\section{Introduction}

A recent article by Wood et al. (2017) demonstrated that both rat and human hepatic in vitro systems (hepatocytes and microsomes) tend to underpredict in vivo intrinsic clearance $\left(\mathrm{CL}_{\mathrm{int}}\right)$ in a clearance-dependent way. The reason for this is not yet clear but in vitro factors including cofactor exhaustion (Swales and Utesch, 1998; Hengstler et al., 2000; Wang et al., 2005) and a rate-limiting unstirred water layer (Wood et al., 2018) may play significant roles. Underprediction of hepatic $\mathrm{CL}_{\mathrm{int}}$ using standard in vitro systems, particularly human, has been recognized for more than a decade but the bias has thus far been generally assumed to be imprecise and irrespective of in vivo $\mathrm{CL}_{\text {int }}$. Over this time, pragmatic empirical correction of bias has frequently been advocated and although this has not been limited to the use of a simple average scaling factor, it has not recognized the clearance dependence (Ito and Houston, 2005; Poulin et al., 2012; Sohlenius-Sternbeck et al., 2012; Yamagata et al., 2017).

In the early 2000 s, it was considered that prediction of clearance from rat hepatocytes was relatively successful compared with the equivalent results from the then emerging use of human hepatocytes (Naritomi et al., 2001, 2003). From this perspective, Naritomi et al. (2003) found that scaling human hepatocyte $\mathrm{CL}_{\text {int }}$ using the single drug observed/predicted $\mathrm{CL}_{\text {int }}$ ratio (single drug empirical scaling factor $\left[\mathrm{ESF}_{\mathrm{sd}}\right]$ ) from rat

This work was supported by the Centre for Applied Pharmacokinetic Research consortium membership, which included Certara, Genentech, Janssen Pharmaceutica, and Eli Lilly \& Co.

https://doi.org/10.1124/dmd.118.085191. hepatocytes improved human predictions to mostly within 5-fold for nine model compounds. They speculated that each compound had an intrinsic scaling factor due to either in vitro nonspecific binding, a lack of equilibrium of blood binding (in vivo), involvement of bound drug in uptake, or heterogeneous distribution of transporters and metabolic enzymes in the liver. Since then, considerable evidence has accumulated confirming that human hepatocytes generally underpredict in vivo $\mathrm{CL}_{\text {int }}$ (Shibata et al., 2002; Hallifax et al., 2005; Ito and Houston, 2005; Riley et al., 2005; Brown et al., 2007; Chiba et al., 2009), attributed to unavoidable donor phenotypic variability, potentially detrimental processing (preparation and storage), and in vitro lability of metabolizing enzymes and uptake transporters, all of which are potential sources of variation and possibly bias. The removal of this bias can be achieved by applying an average hepatocyte-in vivo empirical scaling factor $\left(\mathrm{ESF}_{\mathrm{av}}\right)$ but the lack of any mechanistic basis for this correction limits its use prospectively.

A recent study by De Bruyn et al. (2018) investigated the utility of monkey hepatocytes to improve prediction of human clearance for a range of drugs cleared by hepatic uptake transporters. The authors found that individual drug scaling factors from monkeys applied to human data improved prediction. However, they also found that use of a monkey $\mathrm{ESF}_{\mathrm{av}}$ gave similar improvements in prediction, which confounds the hypothesis that cross-species scaling facilitates common factors related to specific drug properties. This recent attempt at a cross-species approach to maximize human clearance prediction from human hepatocytes has prompted further investigation of the clearance dependence of prediction bias in humans and rats using the dataset of Wood et al. (2017). Our aim

ABBREVIATIONS: $\mathrm{CL}_{\mathrm{int}}$, intrinsic clearance; $\mathrm{ESF}$, empirical scaling factor; $\mathrm{ESF}_{\mathrm{av}}$, average empirical scaling factor; $\mathrm{ESF}_{\mathrm{sd}}$, single drug empirical scaling factor; $E_{S F}$, segregated empirical scaling factor; fub fraction unbound in blood; GMFE, geometric mean fold error; RMSE, root mean squared error. 
was to establish whether the clearance dependence in human hepatic $\mathrm{CL}_{\text {int }}$ prediction from hepatocytes could be corrected by scaling from rat hepatocyte $\mathrm{CL}_{\mathrm{int}}$ based on common system performance; several scaling approaches, including conventional ESFs $\left(\mathrm{ESF}_{\mathrm{av}}, \mathrm{ESF}_{\mathrm{sd}}\right)$ and a novel, multi-ESF approach, were compared (see Table 1).

The use of a cross-species ESF has the potential advantage of being an independent measure of isolated hepatocyte functionality with respect to in vivo activity and hence differs from the conventional ESF, which applies within the same species. Hence it might be used prospectively together with the capacity scalers (hepatocellularity and/or proteomic measurements) to boost confidence in human prediction from either rat or human hepatocytes by way of providing a measure of in vitro functional activity.

\section{Materials and Methods}

Data Source. Datasets for human $(n=101$ for hepatocytes and 83 for microsomes) and rat ( $n=128$ for hepatocytes and 71 for microsomes) in vitro $\mathrm{CL}_{\text {int }}$ and complementary in vivo $\mathrm{CL}_{\text {int }}$ were taken from Wood et al. (2017), in turn based on examination of published datasets, including both approved pharmaceuticals and investigatory proprietary compounds in which blood clearance or plasma clearance $\left(\mathrm{CL}_{\mathrm{p}}\right)$ was determined from intravenous dosing and where blood clearance did not exceed hepatic blood flow. This included in vitro $\mathrm{CL}_{\text {int }}$ determined from either metabolite formation (over a range of substrate concentrations) or from substrate depletion from single substrate concentration depletion time profiles. All hepatocyte data were from suspended cell (fresh or cryopreserved) assays without the addition of serum albumin. Some data were mean values of several independent publications. The datasets were considered to predominantly comprise highly permeable drugs, of which the majority would be expected to be cleared by metabolism without rate limitation by transport. All $\mathrm{CL}_{\text {int }}$ values had been corrected for unbound drug and physiologically scaled to the whole liver using standard methods. The in vivo $\mathrm{CL}_{\text {int }}$ values taken for this study were those calculated from the well stirred liver model, after correction for renal clearance where applicable. The fraction unbound in blood $\left(\mathrm{fu}_{\mathrm{b}}\right)$ was also taken from this dataset.

Correlation Analysis. Predictions of in vivo $\mathrm{CL}_{\mathrm{int}}$ from human hepatocytes were compared with observed in vivo $\mathrm{CL}_{\text {int }}$ for the whole datasets and for the subset of drugs common to both species, as in Wood et al. (2017). The human predicted $\mathrm{CL}_{\text {int }}$ values were compared with corresponding in vivo reported values directly (approach 1) and then corrected using a series of ESFs based on rat predictions of $\mathrm{CL}_{\text {int }}$, as listed in Table 1. First, previously advocated ESFs calculated from rat predictions were applied (approaches 2 and 3 for $\mathrm{ESF}_{\mathrm{av}}$ and $\mathrm{ESF}_{\text {sd }}$, respectively).
To investigate the mechanistic origin of the clearance-dependent bias in prediction, direct comparison between species in vivo (approaches 4 or 5) or the in vitro system (approaches 6-9) was examined using subsets of common drugs within the whole dataset. In addition, approaches 10 and 11 explore correlations between human hepatocyte and human microsome $\mathrm{CL}_{\text {int }}$ (common drugs in humans) and between human hepatocytes and human microsomes from the same donor livers from a specific study (Foster et al., 2011).

Subsequently, a novel prediction correction approach using ESFs based on prediction data segregated according to a particular range of $\mathrm{CL}_{\text {int }}$ values $\left(\mathrm{ESF}_{\mathrm{seg}}\right)$ was examined as a potential means of correcting for clearance-dependent bias. Approaches 12-14 investigate human and rat predictions segregated according to each order of magnitude of observed $\mathrm{CL}_{\text {int }}$ (five levels: $10-10,000 \mathrm{ml} / \mathrm{min} / \mathrm{kg}$ ).

Assessment of Accuracy and Precision of Predictions. The bias in predictions was assessed by calculation of the geometric mean fold error (GMFE) (eq. 1). The root mean squared error (RMSE) (eq. 2) was used as a measure of precision.

$$
\mathrm{GMFE}=10^{\frac{\sum \log \frac{\mathrm{P} r \text { ediced }}{\text { Oberedce }}}{n}}
$$

$$
\text { RMSE }=\sqrt{\frac{1}{n} \sum(\text { Predicted }- \text { Observed })^{2}}
$$

where $n$ is the number of predictions.

Residuals (log 10 predicted/observed) of predictions were examined graphically and the percentage of $\mathrm{CL}_{\text {int }}$ predictions within 2-fold of in vivo was used as an indicator of predictive accuracy, consistent with previous publications (Obach, 1999; Sohlenius-Sternbeck et al., 2012; Chan et al., 2013).

Calculation of ESFs. The ESFs required to equate predicted $\mathrm{CL}_{\text {int }}$ with observed $\mathrm{CL}_{\text {int }}$ for either whole datasets or subsets $\left(\mathrm{ESF}_{\mathrm{av}}\right)$ or for individual compounds $\left(\mathrm{ESF}_{\mathrm{sd}}\right)$ within each dataset were calculated using eqs. 3 and 4 , respectively.

$$
\begin{gathered}
\mathrm{ESF}_{\mathrm{av}}=10^{\frac{\sum \log \frac{\text { Obecerved }}{\text { Prediceded }}}{n}} \\
\mathrm{ESF}_{\mathrm{sd}}=\frac{\text { Observed } \mathrm{CL}_{\text {int,u }}}{\text { Predicted } \mathrm{CL}_{\text {int }, \mathrm{u}}}
\end{gathered}
$$

\section{Results}

Human Hepatocyte In Vitro-In Vivo Correlation. Human in vivo $\mathrm{CL}_{\text {int }}$ was predominantly underpredicted in hepatocytes (Fig. 1, A and B); the average fold-underprediction (GMFE) was 4.2. The overall prediction precision, represented by an RMSE value of

\begin{tabular}{|c|c|c|c|c|c|}
\hline $\begin{array}{l}\text { Approach } \\
\text { Number }\end{array}$ & $\begin{array}{l}\text { System } \\
\text { Comparison }\end{array}$ & Human Parameter $(y)$ & Applied ESF & Human or Rat Parameter $(x)$ & Figure \\
\hline 1 & In vitro-in vivo & Hepatocyte predicted $\mathrm{CL}_{\text {int }}$ & None & Human in vivo $\mathrm{CL}_{\text {int }}$ & $1, \mathrm{~A}$ and $\mathrm{B}$ \\
\hline 2 & & Hepatocyte predicted $\mathrm{CL}_{\text {int }}$ & Rat average $\left(\mathrm{ESF}_{\mathrm{av}}\right)$ & Human in vivo $\mathrm{CL}_{\text {int }}$ & $1, \mathrm{C}$ and $\mathrm{D}$ \\
\hline 3 & & Hepatocyte predicted $\mathrm{CL}_{\text {int }}$ & Rat individual $\left(\mathrm{ESF}_{\mathrm{sd}}\right)$ & Human in vivo $\mathrm{CL}_{\text {int }}$ & $1 \mathrm{E}$ and $\mathrm{F}$ \\
\hline 4 & In vivo-in vivo & $\mathrm{CL}_{\text {int }}$ & N/A & Rat in vivo $\mathrm{CL}_{\text {int }}$ & 2 \\
\hline 5 & & $\mathrm{fu}_{\mathrm{b}}$ & N/A & Rat $f_{b}$ & 3 \\
\hline 6 & In vitro-in vitro & Hepatocyte predicted $\mathrm{CL}_{\text {int }}$ & N/A & Rat hepatocyte predicted $\mathrm{CL}_{\text {int }}$ & $4 \mathrm{~A}$ \\
\hline 7 & & Microsome predicted $\mathrm{CL}_{\text {int }}$ & N/A & Rat microsome predicted $\mathrm{CL}_{\text {int }}$ & 4B \\
\hline 8 & & Hepatocyte absolute $\mathrm{CL}_{\text {int }}$ & N/A & Rat hepatocyte absolute $\mathrm{CL}_{\text {int }}$ & $4 \mathrm{C}$ \\
\hline 9 & & Microsome absolute $\mathrm{CL}_{\text {int }}$ & N/A & Rat microsome absolute $\mathrm{CL}_{\text {int }}$ & 4D \\
\hline 10 & & Hepatocyte predicted $\mathrm{CL}_{\text {int }}$ & N/A & Human microsome predicted $\mathrm{CL}_{\text {int }}$ & $5 \mathrm{~A}$ \\
\hline 11 & & $\begin{array}{l}\text { Hepatocyte predicted } \mathrm{CL}_{\text {int }} \\
\text { (same donor) }\end{array}$ & N/A & $\begin{array}{l}\text { Human microsome predicted } \mathrm{CL}_{\text {int }} \text { (same } \\
\text { donor) }\end{array}$ & $5 \mathrm{~B}$ \\
\hline 12 & In vitro-in vivo & Hepatocyte predicted $\mathrm{CL}_{\text {int }}$ & Rat segregated $\left(\mathrm{ESF}_{\mathrm{seg}}\right)$ & Human in vivo $\mathrm{CL}_{\text {int }}$ & $6 \mathrm{~A}$ \\
\hline 13 & & Hepatocyte predicted $\mathrm{CL}_{\text {int }}$ & Rat $\mathrm{ESF}_{\mathrm{seg}} \times\left[\right.$ human $\left.\mathrm{ESF}_{\mathrm{av}} / \mathrm{rat} \mathrm{ESF}_{\mathrm{av}}\right]$ & Human in vivo $\mathrm{CL}_{\text {int }}$ & $6 \mathrm{C}$ \\
\hline 14 & & Hepatocyte predicted $\mathrm{CL}_{\text {int }}$ & Human $\mathrm{ESF}_{\text {seg }}$ & Human in vivo $\mathrm{CL}_{\text {int }}$ & $6 \mathrm{E}$ \\
\hline
\end{tabular}

TABLE 1

Correlation analysis methods performed for comparison of in vitro-in vivo, in vivo-in vivo, and in vitro-in vitro $\mathrm{CL}_{\mathrm{int}}$ and $\mathrm{f}_{\mathrm{ub}}$ for humans and rats The order shown corresponds to the order of presentation of each correlation in the Results.

N/A, not applicable. 

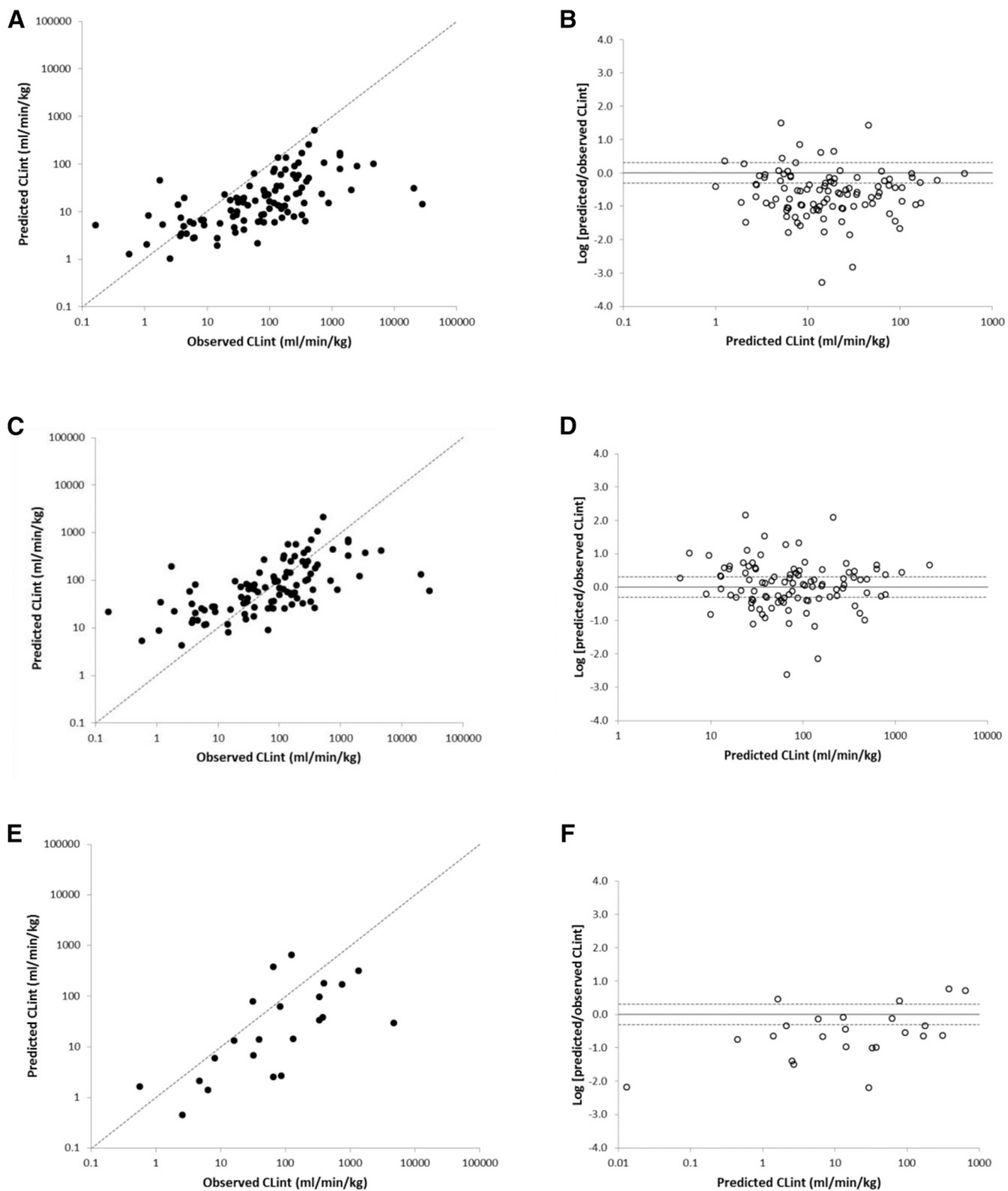

Fig. 1. (A, C, and $\mathrm{E}$ ) Correlation of in vitro $\mathrm{CL}_{\text {int }}$ in human hepatocytes either uncorrected (A), adjusted with average rat scaling factor (C), or adjusted using individual drug rat scaling factors $(\mathrm{E})$ with human in vivo $\mathrm{CL}_{\text {int }}$ for 101 drugs (A and $\mathrm{C}$ ) and 23 drugs (E). Dashed lines represent unity. (B, D, and F) Corresponding residual plots of log predicted/observed $\mathrm{CL}_{\text {int }}$ with predicted $\mathrm{CL}_{\text {int }}$ are shown, respectively.

3550, provides a benchmark against which subsequent predictions, where correction factors are applied, can be compared (Table 2).

As noted previously, microsomes display a wider range of $\mathrm{CL}_{\text {int }}$ predictions than hepatocytes (human and rat); human hepatocyte predicted $\mathrm{CL}_{\text {int }}$ ranged from approximately 1 to $1000 \mathrm{ml} / \mathrm{min} / \mathrm{kg}$ compared with approximately 0.1 to $10,000 \mathrm{ml} / \mathrm{min} / \mathrm{kg}$ in microsomes (Wood et al., 2017). In rats, predicted $\mathrm{CL}_{\text {int }}$ was limited to approximately $10,000 \mathrm{ml} / \mathrm{min} / \mathrm{kg}$ in hepatocytes, whereas it reached $100,000 \mathrm{ml} / \mathrm{min} / \mathrm{kg}$ in microsomes (Wood et al., 2017). Prediction of $\mathrm{CL}_{\text {int }}$ was clearance dependent in both species (and systems), with negative bias increasing 
TABLE 2

Accuracy and precision of human hepatocyte prediction of in vivo $\mathrm{CL}_{\text {int }}$ using rat and human in vitro-in vivo scaling factors, as represented by GMFE, RMSE, and the percentage of predictions that fall within 2 -fold of the observed in vivo $\mathrm{CL}_{\text {int }}$

\begin{tabular}{|c|c|c|c|c|c|c|c|}
\hline \multirow[b]{2}{*}{ Parameter } & \multicolumn{2}{|c|}{ Uncorrected Prediction } & \multicolumn{5}{|c|}{ Corrected Prediction Using a Scaling Factor } \\
\hline & $\begin{array}{l}\text { Total Human } \\
\text { Dataset }\end{array}$ & $\begin{array}{l}\text { Human Drugs } \\
\text { Common to Rats }\end{array}$ & $\begin{array}{c}\text { Rat } \\
\text { Average } \\
\left(\mathrm{ESF}_{\mathrm{av}}\right)\end{array}$ & $\begin{array}{c}\text { Rat } \\
\text { Individual } \\
\text { Drug }\left(\mathrm{ESF}_{\mathrm{sd}}\right)\end{array}$ & $\begin{array}{c}\text { Rat Segregated } \\
\text { Average } \\
\left(\mathrm{ESF}_{\text {seg }}\right)\end{array}$ & $\begin{array}{l}\text { Rat Segregated Average } \times \mathrm{Human} / \text { Rat Ratio } \\
\left(\text { Human } \mathrm{ESF}_{\mathrm{av}} / \mathrm{Rat}^{\mathrm{RSF}} \mathrm{av}_{\mathrm{av}}\right)\end{array}$ & $\begin{array}{c}\text { Human Segregated } \\
\text { Average } \\
\left(\mathrm{ESF}_{\mathrm{seg}}\right)\end{array}$ \\
\hline Rats, $n$ of drugs & N/A & 23 & 128 & 23 & 128 & 128 & 128 \\
\hline Humans, $n$ of drugs & 101 & 23 & 101 & 23 & 101 & 101 & 101 \\
\hline GMFE & 4.20 & 4.35 & 0.896 & 3.82 & 2.82 & 0.802 & 1.00 \\
\hline RMSE & 3550 & 1000 & 3530 & 1010 & 3030 & 2100 & 1990 \\
\hline$\%$ within 2 -fold & 22.8 & N/A & 35.6 & 13.0 & 30.7 & 45.5 & 49.5 \\
\hline Figure & $1 \mathrm{~A}$ & N/A & $1 \mathrm{C}$ & $1 \mathrm{E}$ & $6 \mathrm{~A}$ & $6 \mathrm{C}$ & $6 \mathrm{E}$ \\
\hline
\end{tabular}

N/A, not applicable.

with increasing $\mathrm{CL}_{\text {int }}$. The intercept of the prediction trend line with the line of unity occurred at about $10 \mathrm{ml} / \mathrm{min} / \mathrm{kg}$ for humans and about $100 \mathrm{ml} / \mathrm{min} / \mathrm{kg}$ for rats, signifying generally greater rates in rats compared with humans.

Cross-Species Correction of Bias: Rat-Human Scaling Factors. The similar GMFE prediction bias between humans (4.2; Table 2) and rats (4.7; Wood et al., 2017) indicated potential for consistent cross-species correction for hepatocyte prediction. By applying this simple factor (rat $\mathrm{ESF}_{\mathrm{av}}$ ), bias in human prediction was effectively abolished; the ratcorrected human hepatocyte GMFE was 0.896 (Table 2). However, as expected, precision was virtually unaffected (RMSE $=3530$ ); consequently, clearance-dependent bias remained (Fig. 1, C and D).

Alternatively, using the ESFs for individual drugs $\left(\mathrm{ESF}_{\mathrm{sd}}\right)$, bias was only marginally reduced (GMFE from 4.35 to 3.82 ). For this drugspecific approach, the precision remained about the same (RMSE = 1010) compared with the common drug dataset predictions when no ESF was applied (Fig. 1, E and F; Table 2).

Rat-Human Correlation In Vivo. Comparison of in vivo $\mathrm{CL}_{\text {int }}$ for common drugs between humans and rats revealed that human in vivo $\mathrm{CL}_{\text {int }}$ is on average several-fold less than for rats (Fig. 2), similar to the average bias in human hepatocyte prediction of $\mathrm{CL}_{\text {int }}$ (Table 2). By visual inspection, there was no clearance dependence in this bias, although the dataset was considerably smaller $(n=23)$ than the in vitro-in vivo datasets for all drugs ( $n=101$ for humans and 128 for rats).

There was some discrepancy between human and rat fub although there was no evidence of bias between the species (Fig. 3). Where fu $\mathrm{u}_{\mathrm{b}}$ was less than 0.1 , differences between rats and humans ranged several-fold, indicating potentially considerable differences in $\mathrm{CL}_{\text {int }}$ for highly bound drugs.

Rat-Human Correlation In Vitro. Predicted $\mathrm{CL}_{\text {int }}$ from human hepatocytes was consistently lower than from rat hepatocytes and was clearance dependent (Fig. 4A). Prediction from human microsomes was also consistently lower than from rats; in contrast, there was no clear evidence of clearance dependence (Fig. 4B). More direct assessment of $\mathrm{CL}_{\mathrm{int}}$ between the species at the cellular and subcellular levels was enabled by comparison of unscaled $\mathrm{CL}_{\text {int }}$ (Fig. 4, C and D); in this case, only marginal convergence due to the differences in physiologic scaling factors could be seen and hence the above observations were found to hold.

Human Hepatocyte-Microsome Correlation. Human hepatocyte predictions of $\mathrm{CL}_{\text {int }}$ were marginally lower on average than human microsome predictions, with a tendency for bias to increase with $\mathrm{CL}_{\text {int }}$ (Fig. 5A). This trend was corroborated by a discrete dataset of $\mathrm{CL}_{\mathrm{int}}$ for a series of benzodiazepines in hepatocytes and microsomes from the same livers, from four donors (Foster et al., 2011) (Fig. 5B). In this more specific dataset, hepatocyte bias was less negative for all substrates in those donors showing overall lower $\mathrm{CL}_{\text {int }}$ for all pathways.
Rat-Human Correction of Bias: Clearance-Dependent Scaling Factors. When both the human and rat datasets were segregated into several discrete levels of in vivo $\mathrm{CL}_{\text {int }}$ (Table 3), so that predictions within each human level (one order of magnitude of in vivo $\mathrm{CL}_{\text {int }}$ ) were corrected using the particular equivalent rat $\mathrm{CL}_{\text {int }}$ level average $\mathrm{ESF}\left(\mathrm{ESF}_{\mathrm{seg}}\right)$, clearance dependence was effectively eliminated (Fig. 6, A and B). However, considerable bias remained $(\mathrm{GMFE}=2.82)$ although precision was increased $(\mathrm{RMSE}=3030)$ compared with uncorrected predictions (Table 2). The $\mathrm{ESF}_{\text {seg }}$ values are given in Table 3. To remove the consistent bias, each rat $\mathrm{ESF}_{\text {seg }}$ was multiplied by the ratio of log average human ESF to log average rat ESF; this two-step scaling approach achieved effective elimination of bias (GMFE $=0.802$; Fig. 6, E and F).

In comparison, using human $\mathrm{ESF}_{\text {seg }}$ to correct human predictions completely eliminated the bias, as expected (GMFE $=1.00)$ (Fig. 6, C and D). The precision was greater than any other method applied to this dataset $(\mathrm{RMSE}=1990)$, providing a representation of the optimum precision obtainable by this kind of empirical correction for this particular dataset. The precision achieved using the rat $\mathrm{ESF}_{\text {seg }}$ (together with the ratio of human $\mathrm{ESF}_{\mathrm{av}} / \mathrm{rat} \mathrm{ESF}_{\mathrm{av}}$, as above) was similar, with an RMSE of 2100.

\section{Discussion}

It has become clear in recent years that physiologic scaling of $\mathrm{CL}_{\text {int }}$ obtained from suspended human hepatocytes not only underpredicts in vivo $\mathrm{CL}_{\text {int }}$ on average (Ito and Houston, 2005; Riley et al., 2005;

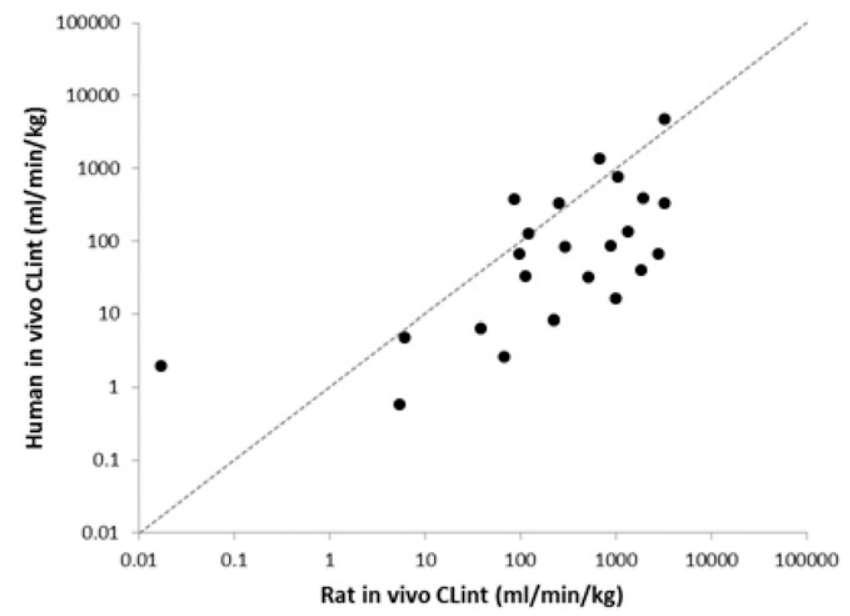

Fig. 2. Correlation of human and rat in vivo $\mathrm{CL}_{\text {int }}$ for 23 drugs. Dashed lines represent unity. 


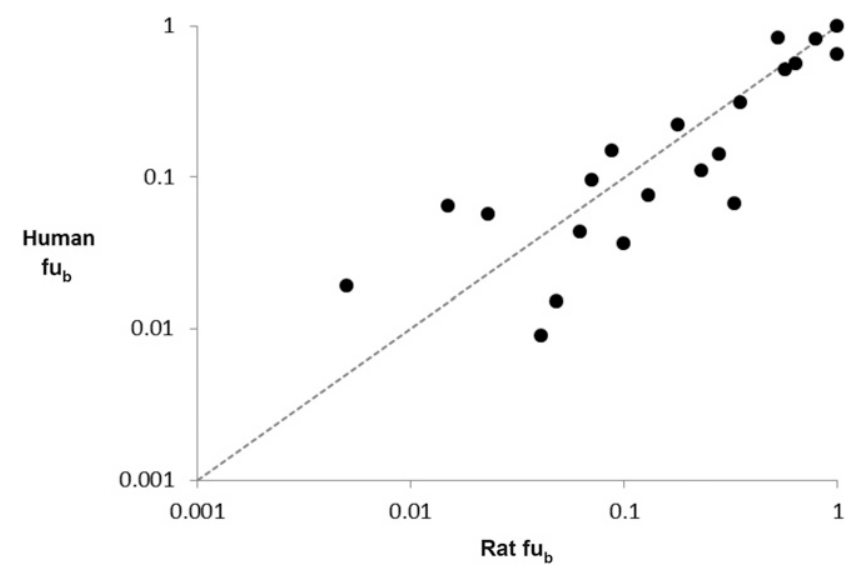

Fig. 3. Correlation of rat and human $\mathrm{fu}_{\mathrm{b}}$ for 23 drugs. Dashed lines represent unity.

Brown et al., 2007; Bowman and Benet, 2016) but does so in a clearance-dependent way (Hallifax et al., 2010; Wood et al., 2017). Although the reasons for this dependence remain unclear but could involve effects of the unstirred water layer and/or cofactor depletion in vitro (Hengstler et al., 2000; Hewitt et al., 2000; Hewitt and Utesch, 2004; Hallifax et al., 2010; Foster et al., 2011; Wood et al., 2017, 2018), achieving resolution of this clearance-dependent bias from the considerable prediction uncertainty has provided an opportunity to apply better targeted empirical correction. Because prediction from rat hepatocytes has now been shown to be clearance dependent in the same manner as human hepatocytes (Wood et al., 2017), there appears to exist a basis for renewed potential in the utility of rat hepatocytes for prediction of human in vivo $\mathrm{CL}_{\text {int }}$.

The main purpose of this study was to compare conventional ESFs between rat and human hepatocyte $\mathrm{CL}_{\text {int }}$ with a novel ESF scaling approach to deal with clearance dependence. At the same time, we examined the relationships between rat and human $\mathrm{CL}_{\text {int }}$ both in vivo and in vitro and between human hepatocytes and microsomes for further mechanistic insight into species-dependent and speciesindependent performance in vitro. This might offer a means of empirical enhancement of conventional scaling that would take account of factors affecting inherent functional activity in vitro, in addition to physiologic capacity.

For these datasets comprising drugs primarily cleared by metabolism without permeability limitation, use of the rat average ESF clearly removes the average bias for human prediction but without increasing precision, leaving unsatisfactory prediction uncertainty. Despite the considerable imprecision among the predictions, clearance dependence was previously resolved (Hallifax et al., 2010; Wood et al., 2017); as such, it is clear that a single scaling factor is inappropriate.
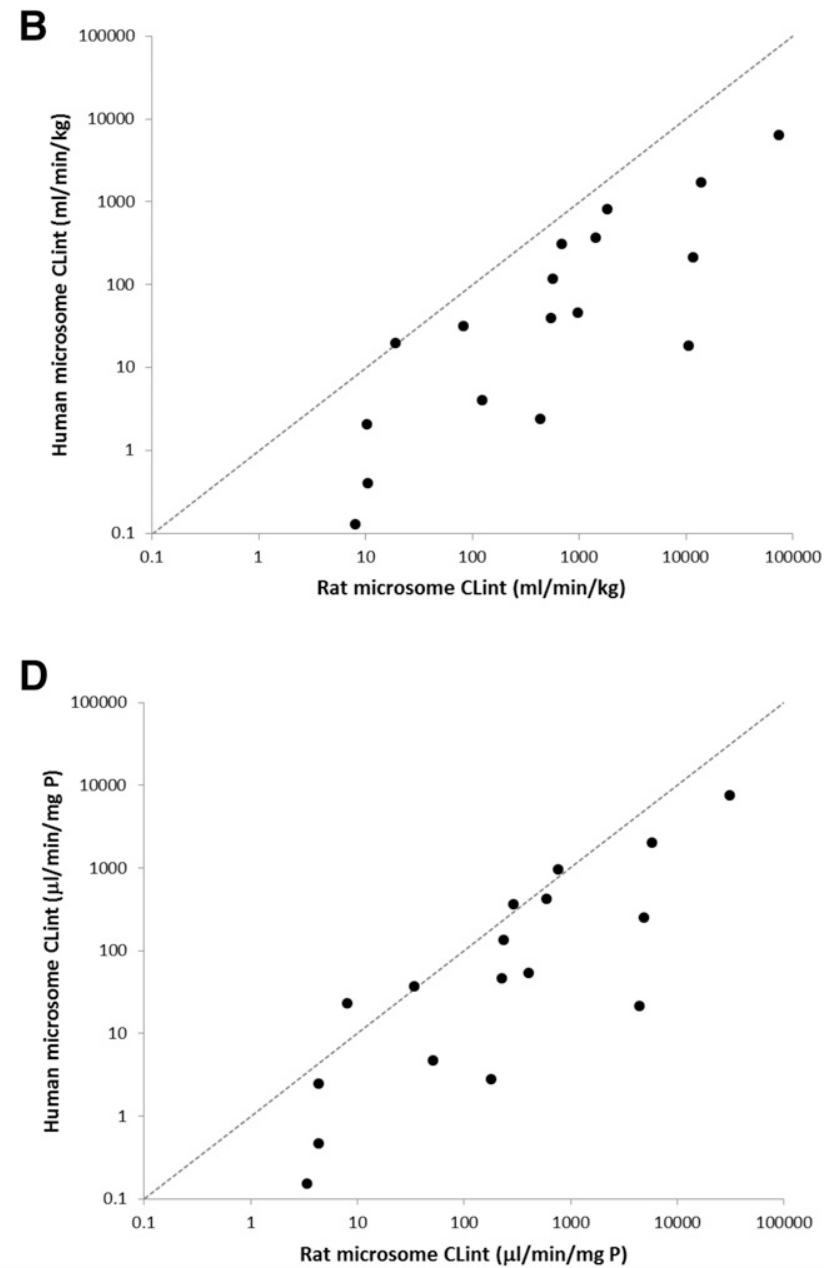

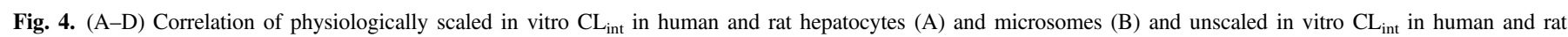
hepatocytes (C) and microsomes (D). Dashed lines represent unity. 
A

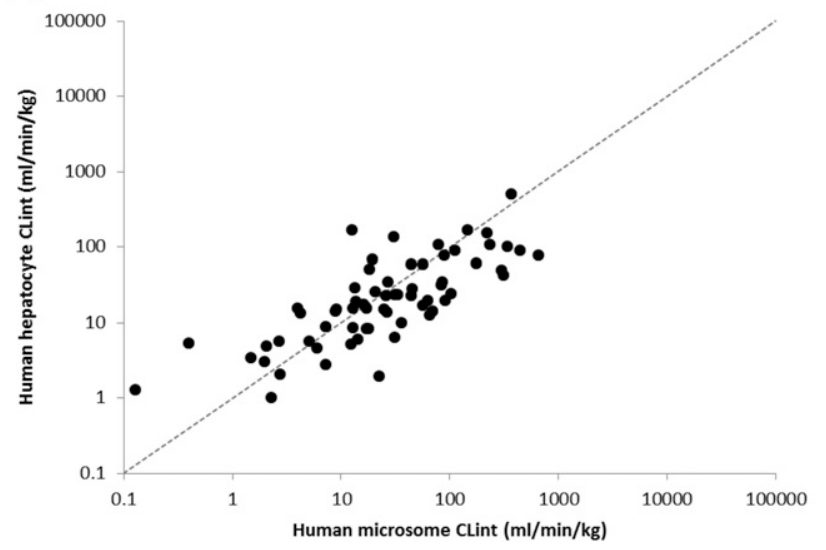

B

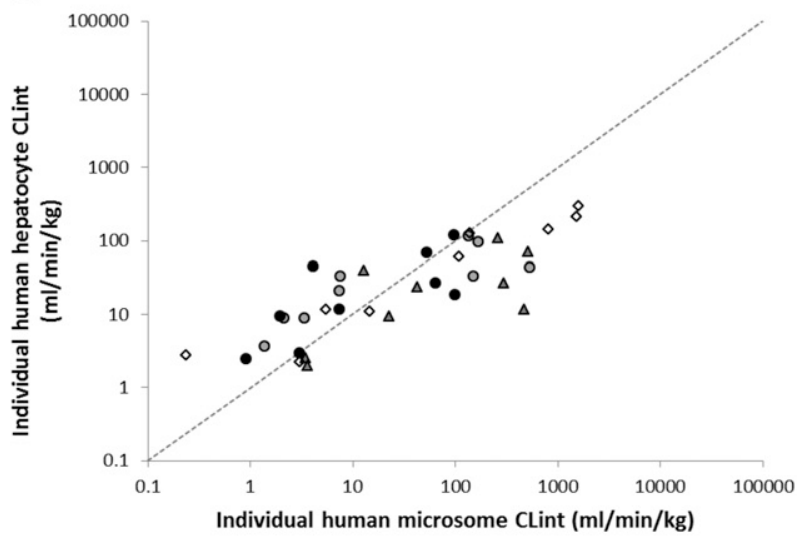

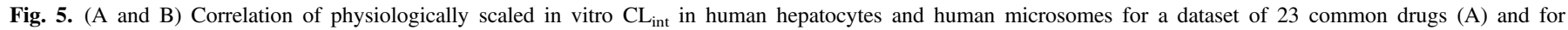
hepatocytes and microsomes from the same donor livers (B), where different symbols represent the different donors. Dashed lines represent unity.

Scaling factors for individual drugs is a concept that gained some traction after studies by Naritomi et al. (2001, 2003) showed prediction improvement (from 12- to 199-fold to within about 5-fold for hepatocytes), which was attributed to drug-specific factors. Yet according to the literature, this approach does not appear to have become widely adopted; rather, various groups have pursued a variety of alternative, empirical, and semi-mechanistic approaches to the problem of underprediction of clearance (Sohlenius-Sternbeck et al., 2012; Yamagata et al., 2017). Wood et al. (2017) examined literature prediction datasets for common drugs between humans and rats (as used in our study) and found no correlation of individual ESFs between rats and humans, which does not support a drug-specific premise. Using the same data, we examined the relationship between human and rat hepatocyte predictions rather than individual ESFs; although there was a correlation, which was biased in favor of the rat, it was clearance dependent. Thus there remains little evidence that drug-specific ESFs offer an improvement in prediction uncertainty. This agrees with recent findings for prediction of transportermediated clearance, in which the application of individual drug ESFs from monkey hepatocytes to human hepatocyte $\mathrm{CL}_{\text {int }}$ did not markedly improve prediction of in vivo clearance compared with the use of an average monkey hepatocyte ESF (De Bruyn et al., 2018). It is apparent that other factors in the in vitro-scaling processes need to be considered and resolved.

The subset of predictions for drugs common to both human and rat hepatocyte predictions showed that in vitro $\mathrm{CL}_{\text {int }}$ tends to be greater in rats by several-fold, although with considerable imprecision, similar to in vitro-in vivo predictions. From a broad interspecies scaling perspective, it is expected that rat hepatic clearance exceeds that of humans due to greater liver relative volume and greater relative hepatic blood flow. However, when focusing specifically on the $\mathrm{CL}_{\text {int }}$

TABLE 3

Segregated scaling factors for rat and human hepatocytes as given by log observed/predicted $\mathrm{CL}_{\text {int }}$

\begin{tabular}{llllll}
\hline \multirow{2}{*}{ Species } & \multicolumn{5}{c}{ Log average Observed/Predicted for Segregated Observed $\mathrm{CL}_{\text {int }}$} \\
\cline { 2 - 6 } & $<10$ & $10-100$ & $100-1000$ & $1000-10,000$ & $>10,000$ \\
\hline \multirow{5}{*}{ Human } & 0.609 & 3.94 & $\mathrm{ml} / \mathrm{min} / \mathrm{kg}$ \\
Rat & 0.125 & 1.57 & 3.24 & 22.2 & 1150 \\
Human/rat & 4.88 & 2.50 & 2.20 & 3.17 & 179 \\
& & & \multicolumn{5}{c}{6.46} \\
\hline
\end{tabular}

parameter, which is normalized to hepatic blood flow, the remaining interspecies discrepancy would signify that the rat liver is inherently more efficient at clearing drugs than its human counterpart. As apparent in this study, human hepatocyte $\mathrm{CL}_{\text {int }}$ is less than that of rats in a clearance-dependent way; at high $\mathrm{CL}_{\text {int }}(>100 \mathrm{ml} / \mathrm{min} / \mathrm{kg})$, there is a tendency toward a more than 10-fold difference between the species. However, while the generally lower $\mathrm{CL}_{\mathrm{int}}$ in human hepatocytes is reflected in the equivalent comparison for microsomes, there does not appear to be a clearance-dependent relationship in this subcellular system. Therefore, a consistent difference between rat and human hepatic metabolic $\mathrm{CL}_{\text {int }}$ (of several-fold) is indicated. This appears to reflect the general difference in in vivo $\mathrm{CL}_{\text {int }}$ between the species, suggesting a generally greater capacity and/or affinity of drugmetabolizing enzymes in rat hepatocytes. As hepatocytes from both species give clearance-dependent prediction (Wood et al., 2017), the consistency in microsomes indicates the existence of both a speciesdependent factor (difference in metabolic efficiency) and a speciesindependent factor underlying a relatively greater inability of human hepatocytes to process high-clearance drugs compared with rat hepatocytes. Thus, the interspecies examination described appears to resolve factors underlying the performance of hepatocytes in vitro, despite the uncertainty recognized among predictions, and this may support attempts at a general, empirical approach to address clearance dependence in prediction.

Despite the complication of clearance-dependent prediction from human and rat hepatocytes, this whole-cell in vitro system should remain the preferred choice compared to hepatic microsomes. In addition to accommodating a more comprehensive set of metabolic pathways than microsomes (Engtrakul et al., 2005; Riley et al., 2005; Hallifax et al., 2010), hepatocytes allow potentially influential uptake as well as the overall disposition processes to be assessed, which is increasingly important given trends in drug discovery toward larger molecules with limited permeability. To address the clearance dependence in prediction (at least empirically), it is clear that ESFs need to be clearance dependent as well. In addition, the possibility emerges that such scaling factors from the rat, as a key preclinical species, can be applied to the human situation prospectively for improved prediction among sets of test compounds. To this end, a panel of segregated rat ESFs $\left(\mathrm{ESF}_{\text {seg }}\right)$ was calculated based on the separation of paired predicted and observed values, according to different ranges of observed $\mathrm{CL}_{\text {int }}$. A relatively simple segregation, by order of magnitude of observed $\mathrm{CL}_{\mathrm{int}}$, was found adequate to achieve effective removal of clearance dependence. Although bias between 


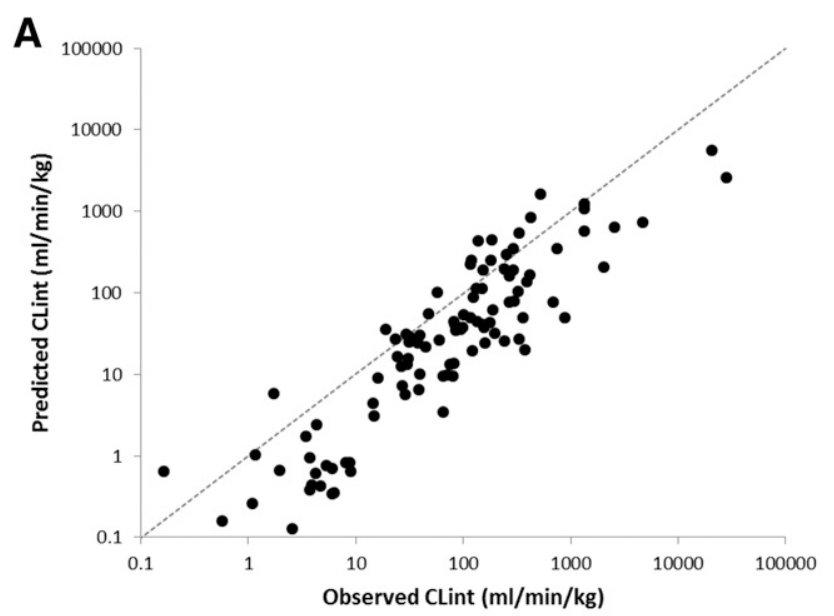

B
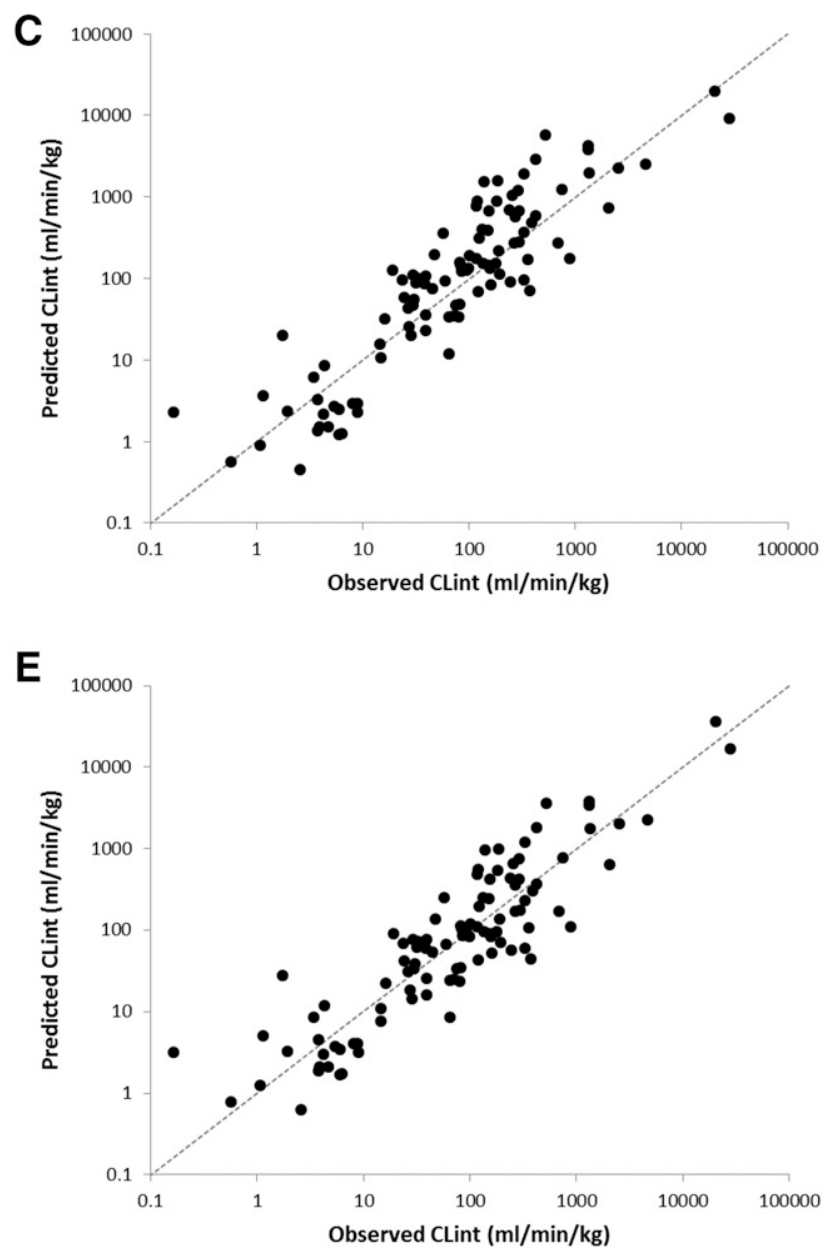

Fig. 6. (A, $\mathrm{C}$, and $\mathrm{E}$ ) Correlation of in vitro $\mathrm{CL}_{\text {int }}$ in human hepatocytes adjusted using rat scaling factors for segregated in vivo $\mathrm{CL}_{\text {int }}(\mathrm{A})$, adjusted using rat scaling factors for segregated in vivo $\mathrm{CL}_{\mathrm{int}}$ multiplied by log average segregated human $\mathrm{ESF} / \log$ average segregated rat $\mathrm{ESF}(\mathrm{C})$, or using human scaling factors for segregated in vivo $\mathrm{CL}_{\mathrm{int}}$ (E). Dashed lines represent unity. (B, D, and F) Corresponding residual plots of log predicted/observed $\mathrm{CL}_{\text {int }}$ with predicted $\mathrm{CL}_{\text {int }}$ are shown, respectively.

the species remained, this was now relatively consistent, reflecting some improvement in precision. Having removed the clearance dependence, comparison of $\mathrm{ESF}_{\text {seg }}$ between rats and humans by $\mathrm{CL}_{\text {int }}$ level revealed a relatively constant cross-species factor for hepatocytes, as anticipated in the earlier species comparisons. Therefore, a second $\mathrm{ESF}$ (human $\mathrm{ESF}_{\mathrm{av}} / \mathrm{rat} \mathrm{ESF}_{\mathrm{av}}$ ) was introduced to correct this apparently inherent difference in metabolic rate between the two
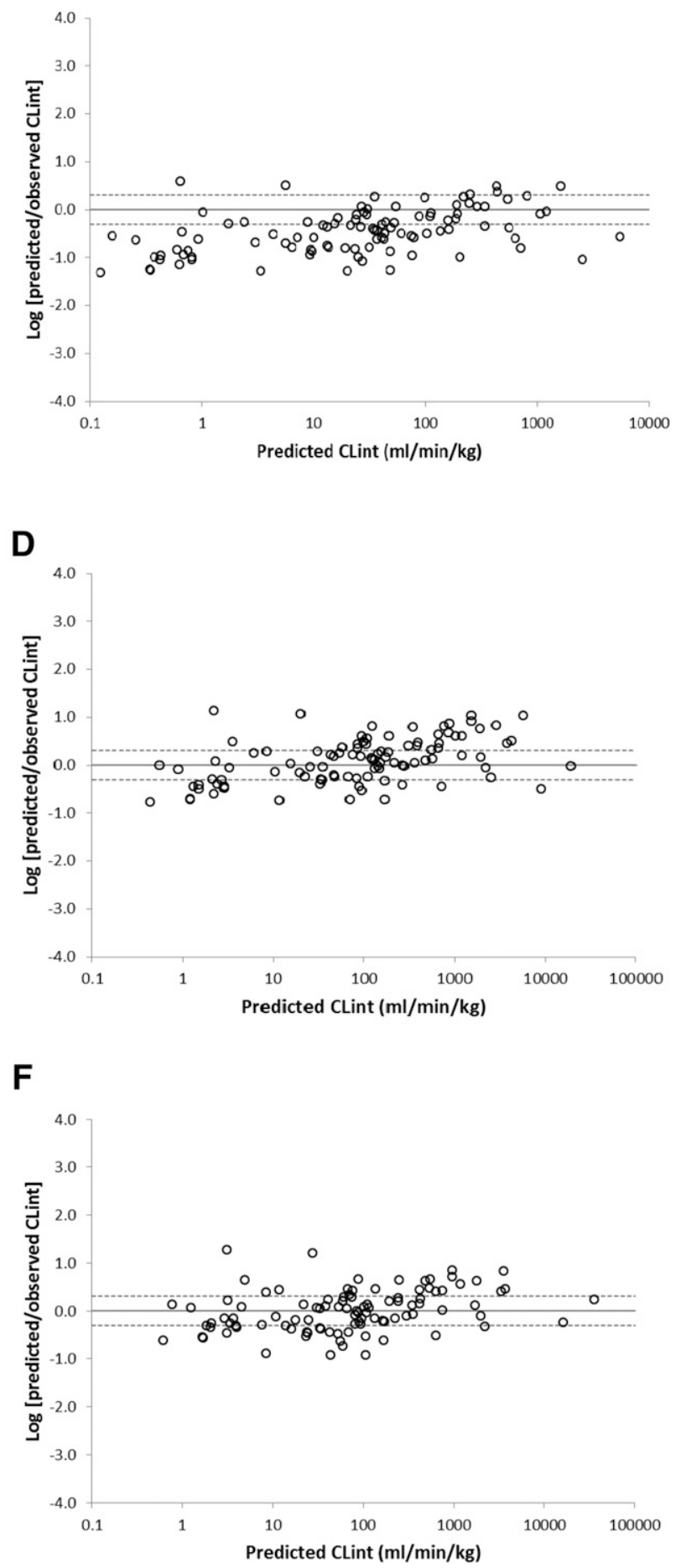

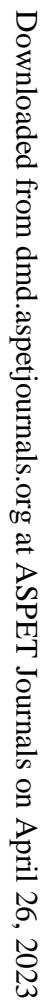

species. By applying these ESFs in sequence, human hepatocyte predictions became largely free of bias with improved precisionvirtually identical to the human internally corrected predictions. Hence, a simple basis for cross-species scaling was established that offers an overall improvement of predictions of human $\mathrm{CL}_{\text {int }}$ from rat hepatocyte $\mathrm{CL}_{\text {int }}$. For these drugs (metabolically cleared without permeability limitation), it can be seen that a human ESF at one 
particular level of $\mathrm{CL}_{\mathrm{int}}$ is approximately matched by a rat $\mathrm{ESF}$ at the $\mathrm{CL}_{\text {int }}$ level one order of magnitude greater. This displacement appears to equal an interspecies factor of about 3-fold (as discussed above), which when factored by the clearance-dependent $\mathrm{ESF}\left(\mathrm{ESF}_{\mathrm{seg}}\right)$ allows consistent correction between these two species. Therefore, sets of predictions of human $\mathrm{CL}_{\text {int }}$ via rat hepatocytes might be more confidently undertaken using the rat scaling factors described above, in addition to the direct use of human $\mathrm{ESF}_{\text {seg }}$ to human hepatocyte $\mathrm{CL}_{\text {int }}$. While it is tempting to assume that $\mathrm{CL}_{\text {int }}$ for any individual drug in rats can be successfully scaled to the human situation by this factor, the observation that specific drug hepatocyte $\mathrm{CL}_{\text {int }}$ appears not to be linearly related between humans and rats means that a species differential may not hold consistently for individual drugs.

When $\mathrm{CL}_{\text {int }}$ was directly compared between hepatocytes and microsomes from the same human liver donors, there was a tendency for $\mathrm{CL}_{\text {int }}$ in both systems to be relatively low or high between individuals (Foster et al., 2011). Because this phenomenon holds for low and high $\mathrm{CL}_{\text {int }}$ pathways for the same drug, a consistent liver factor may be invoked that feeds through to a difference between hepatocyte and microsome $\mathrm{CL}_{\mathrm{int}}$ according to the level of $\mathrm{CL}_{\mathrm{int}}$. For individuals with relatively high hepatic activity compared with others, there is thus a tendency for the highest $\mathrm{CL}_{\text {int }}$ pathways to give lower $\mathrm{CL}_{\text {int }}$ in hepatocytes compared with microsomes (and vice versa). Therefore, it appears that the $\mathrm{CL}_{\text {int }}$ level, rather than the individual drug, is the driver of this scaling phenomenon. Notwithstanding inevitable contributions from drug-specific factors, a predominantly system-specific phenomenon would explain why drug-specific ESFs $\left(\mathrm{ESF}_{\mathrm{sd}}\right)$ are not particularly successful, as alluded to above. It is also notable that this relationship prevails at low $\mathrm{CL}_{\text {int }}$ where for human prediction $(<10 \mathrm{ml} / \mathrm{min}$ per kilogram), the incidence of underprediction is more or less negated by the incidence of overprediction (in terms of bias). The reasons for this system-specific scaling phenomenon are not clear, although some difference in metabolic capacity between rat and human hepatocytes is indicated in this study.

Beyond the system-specific factor, there are species differences between hepatocytes whose impact can be dependent on the drug, such as the shaking of hepatocyte incubations (Wood et al., 2018). Other drugspecific species differences in hepatic clearance include metabolic and transporter pathways, not least due to species differences in cytochrome $\mathrm{P} 450$ enzymes or transporters resulting in differences in capacity and/or affinity. In addition, differences in the extent of binding to plasma proteins could reflect significant species differences in protein. Hence, there is potential for recategorization of a drug's clearance level between rats and humans and factors specific between drug- and species-specific proteins may further explain why drug-specific ESFs are not particularly successful. In addition, experimental methodology may confound species comparison, including failure to identify high-affinity clearance pathways due to inadequately low substrate concentration (Klopf and Worboys, 2010; Wood et al., 2018).

The relationship between human and rat $\mathrm{CL}_{\text {int }}$ both in vitro and in vivo has been explored for historical datasets of drugs comprising drugs predominantly cleared by metabolism without permeability limitation and, based on the species-dependent and species-independent factors revealed, viable empirical improvement of human $\mathrm{CL}_{\mathrm{int}}$ prediction from either human or rat hepatocytes is indicated. Nevertheless, further experimental examination of the sources of uncertainty are advocated, including potentially influential drug-specific uncertainties, due to the need for more accurate in vitro pharmacokinetic parameter values for confident physiologically based pharmacokinetic modeling.

\section{Authorship Contributions}

Participated in research design: Hallifax, Houston.
Performed data analysis: Hallifax, Houston.

Wrote or contributed to the writing of the manuscript: Hallifax, Houston.

\section{References}

Bowman CM and Benet LZ (2016) Hepatic clearance predictions from in vitro-in vivo extrapolation and the biopharmaceutics drug disposition classification system. Drug Metab Dispos 44:1731-1735.

Brown HS, Griffin M, and Houston JB (2007) Evaluation of cryopreserved human hepatocytes as an alternative in vitro system to microsomes for the prediction of metabolic clearance. Drug Metab Dispos 35:293-301

Chan TS, Yu H, Moore A, Khetani SR, and Tweedie D (2013) Meeting the challenge of predicting hepatic clearance of compounds slowly metabolized by cytochrome P450 using a novel hepatocyte model, HepatoPac [published correction appears in Drug Metab Dispos (2014) 42:200 and Drug Metab Dispos (2019) 47:58-66]. Drug Metab Dispos 41:2024-2032.

Chiba M, Ishii Y, and Sugiyama Y (2009) Prediction of hepatic clearance in human from in vitro data for successful drug development. AAPS J 11:262-276.

De Bruyn T, Ufuk A, Cantrill C, Kosa RE, Bi YA, Niosi M, Modi S, Rodrigues AD, Tremaine LM, Varma MVS, et al. (2018) Predicting human clearance of organic anion transporting polypeptide substrates using cynomolgus monkey: in vitro-in vivo scaling of hepatic uptake clearance. Drug Metab Dispos 46:989-1000.

Engtrakul JJ, Foti RS, Strelevitz TJ, and Fisher MB (2005) Altered AZT (3'-azido-3'-deoxythymidine) glucuronidation kinetics in liver microsomes as an explanation for underprediction of in vivo clearance: comparison to hepatocytes and effect of incubation environment. Drug Metab Dispos 33:1621-1627.

Foster JA, Houston JB, and Hallifax D (2011) Comparison of intrinsic clearances in human liver microsomes and suspended hepatocytes from the same donor livers: clearance-dependent relationship and implications for prediction of in vivo clearance. Xenobiotica 41:124-136.

Hallifax D, Foster JA, and Houston JB (2010) Prediction of human metabolic clearance from in vitro systems: retrospective analysis and prospective view. Pharm Res 27:2150-2161.

Hallifax D, Rawden HC, Hakooz N, and Houston JB (2005) Prediction of metabolic clearance using cryopreserved human hepatocytes: kinetic characteristics for five benzodiazepines. Drug Metab Dispos 33:1852-1858.

Hengstler JG, Utesch D, Steinberg P, Platt KL, Diener B, Ringel M, Swales N, Fischer T, Biefang $\mathrm{K}$, Gerl M, et al. (2000) Cryopreserved primary hepatocytes as a constantly available in vitro model for the evaluation of human and animal drug metabolism and enzyme induction. Drug Metab Rev 32:81-118.

Hewitt NJ, Fischer T, Zuehlke U, Oesch F, and Utesch D (2000) Metabolic activity of fresh and cryopreserved cynomolgus monkey (Macaca fascicularis) hepatocytes. Xenobiotica 30:665-681. Hewitt NJ and Utesch D (2004) Cryopreserved rat, dog and monkey hepatocytes: measurement of drug metabolizing enzymes in suspensions and cultures. Hum Exp Toxicol 23:307-316.

Ito $\mathrm{K}$ and Houston JB (2005) Prediction of human drug clearance from in vitro and preclinical data using physiologically based and empirical approaches. Pharm Res 22:103-112.

Klopf W and Worboys P (2010) Scaling in vivo pharmacokinetics from in vitro metabolic stability data in drug discovery. Comb Chem High Throughput Screen 13:159-169.

Naritomi Y, Terashita S, Kagayama A, and Sugiyama Y (2003) Utility of hepatocytes in predicting drug metabolism: comparison of hepatic intrinsic clearance in rats and humans in vivo and in vitro. Drug Metab Dispos 31:580-588.

Naritomi Y, Terashita S, Kimura S, Suzuki A, Kagayama A, and Sugiyama Y (2001) Prediction of human hepatic clearance from in vivo animal experiments and in vitro metabolic studies with liver microsomes from animals and humans. Drug Metab Dispos 29:1316-1324.

Obach RS (1999) Prediction of human clearance of twenty-nine drugs from hepatic microsomal intrinsic clearance data: an examination of in vitro half-life approach and nonspecific binding to microsomes. Drug Metab Dispos 27:1350-1359.

Poulin P, Kenny JR, Hop CECA, and Haddad S (2012) In vitro-in vivo extrapolation of clearance: modeling hepatic metabolic clearance of highly bound drugs and comparative assessment with existing calculation methods. J Pharm Sci 101:838-851.

Riley RJ, McGinnity DF, and Austin RP (2005) A unified model for predicting human hepatic, metabolic clearance from in vitro intrinsic clearance data in hepatocytes and microsomes. Drug Metab Dispos 33:1304-1311.

Shibata Y, Takahashi H, Chiba M, and Ishii Y (2002) Prediction of hepatic clearance and availability by cryopreserved human hepatocytes: an application of serum incubation method. Drug Metab Dispos 30:892-896.

Sohlenius-Sternbeck AK, Jones C, Ferguson D, Middleton BJ, Projean D, Floby E, Bylund J, and Afzelius L (2012) Practical use of the regression offset approach for the prediction of in vivo intrinsic clearance from hepatocytes. Xenobiotica 42:841-853.

Swales NJ and Utesch D (1998) Metabolic activity of fresh and cryopreserved dog hepatocyte suspensions. Xenobiotica 28:937-948.

Wang Q, Jia R, Ye C, Garcia M, Li J, and Hidalgo IJ (2005) Glucuronidation and sulfation of 7-hydroxycoumarin in liver matrices from human, dog, monkey, rat, and mouse. In Vitro Cell Dev Biol Anim 41:97-103.

Wood FL, Houston JB, and Hallifax D (2017) Clearance prediction methodology needs fundamental improvement: trends common to rat and human hepatocytes/microsomes and implications for experimental methodology. Drug Metab Dispos 45:1178-1188.

Wood FL, Houston JB, and Hallifax D (2018) Importance of the unstirred water layer and hepatocyte membrane integrity in vitro for quantification of intrinsic metabolic clearance. Drug Metab Dispos 46:268-278.

Yamagata T, Zanelli U, Gallemann D, Perrin D, Dolgos H, and Petersson C (2017) Comparison of methods for the prediction of human clearance from hepatocyte intrinsic clearance for a set of reference compounds and an external evaluation set. Xenobiotica 47:741-751.

Address correspondence to: D. Hallifax, Centre for Applied Pharmacokinetic Research, Division of Pharmacy and Optometry, School of Health Sciences, Faculty of Biology, Medicine and Health, Manchester Academic Health Science Centre, University of Manchester, Manchester M13 9PT, UK. E-mail: david. hallifax@manchester.ac.uk 